\title{
EEG Waves for Robotics and Prosthesis Grasping and Motorization
}

\author{
Ebrahim A. Mattar
}

\begin{abstract}
Dexterous multi-finger robotics hands/or prosthesis hands are complicated devices to model, control, and to motorize. Modeling involves building coordinated kinematics relations, while the dynamic model involves grasping forces and optimized distributions of forces and torques at grasping locations. Over the last thirty years or more of research, a coordinated control of fingers for such devices was done analytically, however such control issues were facing few number of difficulties. Therefore, the purpose of this paper is to look at novel approach for defining grasping patters from EEG readings, then to learn-mirror such patters into robotics handprosthesis. We shall create an association between fingers motions, forces, and particularly detected EEG brainwaves from human. Such an association is very useful for robotics humanoids, or for prosthesis. The association between human EEG to robotics is modeled here, and it will be used for grasping by system robotic by learning (via training) a robotics multi-finger dexterous hands. In addition, such an association is also useful for controlling a prosthesis for rehabilitations purposes.
\end{abstract}

Index Terms-EEG, rehabilitation, BMI, robotic-prosthetic, patterns recognition, learning systems.

\section{INTRODUCTION}

\section{A. Robotics and Rehabilitation}

Grasping with five fingers by a robotics or prosthesis is not an easy task. Two issues are to be considered, the first is related to finger-digits coordinated motion, while the second is totally related to how fingertips forces are applied and manipulated. Within this research, we shall be presenting a methodology for using human EEG brainwaves patterns for decoding, analysis, train, hence to motorize robotics hand/prosthesis for grasping purposes. In particular, we try to associate the recorded brainwaves patterns with the various five finger motions, and fingertips forces, Fig. 1. In this respect, dividing and clustering of hand EEG grasping patterns do represent to focal problem to be solved, Fig. 2.

"Stroke is defined as 'rapidly developing clinical signs of focal (at times global) disturbance of cerebral function, with symptoms lasting 24 hours or longer or leading to death, with no apparent cause other than of vascular origin' ([1], Hatano)". Within same respect, there has been a number of studies and research about robotics and their use for rehabs applications, where it was found that, practicing such an intense and active repeated hand and fingers practice is to

Manuscript received March 21, 2016; revised June 25, 2016.

E. A. Mattar is with the College of Engineering, University of Bahrain, Sukhair, P.O. Box 32038, Kingdom of Bahrain (e-mail ebmattar@uob.deu.bh). enhance the functionalities of affected arm and hand. Refer to [2]-[8] for further works and analysis in this respect.

Loss of hand function causes a severe decrease in quality of life for affected individuals [9]. In addition, there have been different classes of therapies. However, lasting disabilities result in a typical lifetime cost between $\$ 100 \mathrm{k}$ and $\$ 2 \mathrm{M}$ per patient, including inpatient care, rehabilitation, and follow-up. The most effective therapies have patients actively controlling their limb, which is not an option in cases of severe paralysis. Through intensive and effective therapies, it was found that most of the patients are able to control and move their limbs, however, this is not possible once the patient is suffering from severe paralysis. Over the last number of years, it has been reported that, BCIs are helpful in terms of picking up brain signals and associated technology to do so, are developing very fast.

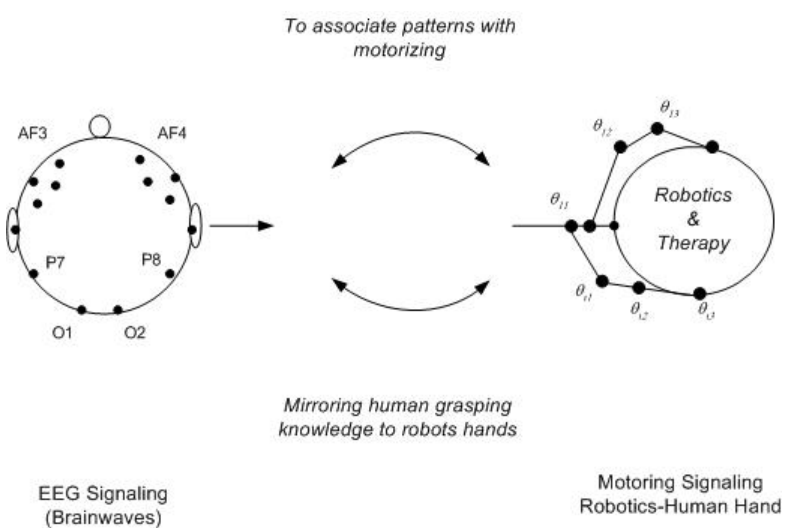

Robotics-Human Hand

Fig. 1. Associating EEG patterns to fingertips motorizing.

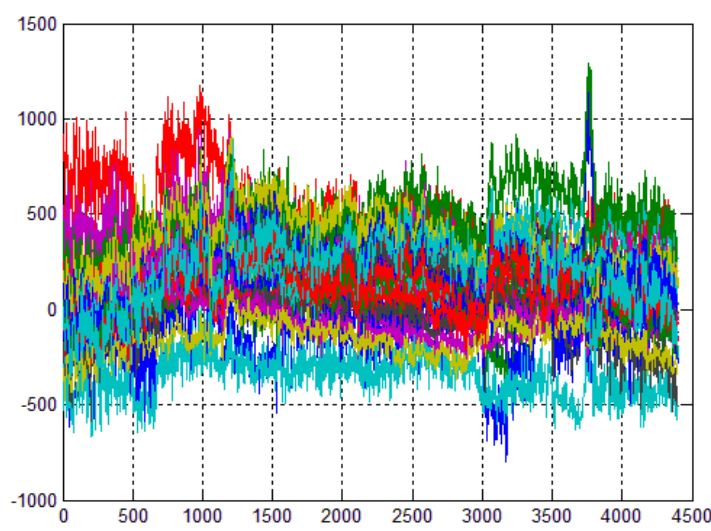

Fig. 2. Typical 32- channels EEG recorded patterns for a grasping trial, [10], [11], [14]

While BMI involves a computational stages, resulting EEG as in Fig. 2, BMI promises new hope for treatment, they remain in the research stage. In addition, conventional BMIs cannot be applied so easily, and specifically once it comes to cases of brain injury since the classical motor signals in cortex 
contralateral to the target limb needed would be gone with the injury. In addition, detecting the brain waves signals through inserting the Utah Electrodes (via surgery) is not a straight forward procedure. There are a number of recent studies related to human hand fingers motion in relation to specific brain wave signals. Of a particular important is the hand finger motion, and the manner human applies forces during different grasping situations. Robotic-Prosthesis control, and their rehabilitation applications are getting active in terms of research trends and tools. Re-motorization of hand fingers after brain caused disabilities is not an obvious task. In addition, conventional neurological therapies and rehabilitations efforts, have been found ineffective in rehabilitating upper-limb function after stroke or even traumatic brain injuries. With the advances of brain interfacing technologies, this made it possible to restore some of the motor functionalities. Advances in technology has resulted in new developments to help people with severe paralysis or even with limb loss. Intracortical brain computer interfaces, are also being developed to enable personals. Recently, there are tremendous efforts and research directions to use the EEG Brain waves signals and their associated patterns for Robotic-Prosthesis applications. This includes the rehabilitations (Rehab) applications. However, due to the complexity of such brain patterns, making use of such complex patterns for practical grasping learning Robotic-Prosthesis is not a trivial task. In this context, this talk will be focusing on decoding and the use of the learned patterns of neural waves neural waves neural waves for grasping applications, in addition to motoring and motion control. This is based on using BMI (Brain Machine Interface) tools, hence to associate such waves for motorizing and controlling a Robotic- prosthesis. In addition, the talk will be focusing on dealing with massive interrelated waves, and how PCA learning patterns are used for such learning. At the end, the talk will also present the current trends world-wide within such active area of research.

Within this paper and in reference to Fig. 3, we shall be addressing the problem of relating BNI technology to rehabilitation and motion restoration for hand-fingers motion, as following stroke and traumatic brain injuries, or even other related brain dysfunctional behavior that prevent motoring of fingers. Some previous efforts in a similar direction, they have employed the UTAH Prop to detect brain waves. However, such an approach does require an insertion of UTAH prop legs beneath the skulls. A fundamental work to be presented is the using other brain wave sensing techniques. In order to achieve that, we shall rely on a developed brain wave signal detection devices (like the massive signals head cap) for brain waves detection rehabilitation that synthesizes recent developments in neurophysiology, electronics, and physical therapy into a BCI hand orthosis. Such brain waves signals were present in cortex anterior to ipsilateral primary motor cortex, with wave patterns operating below 40Hz [12], [13]. Such patterns are accessible via EEG.

Once brain waves signals are detected and conditioned, they are then analyzed for pattern recognition via artificial neural network. Models are built associated with such signals. Finally a closed control system is built around the artificial limb (the hand), to restore the motoring action. Limb (Hand) tactile sensing and closing the loop in a feedback will facilitate neural plasticity. In addition it will be strengthening existing and developing new neural pathways ipsilateral to the affected hand that will ultimately restore motor control. Allowing the patient to regain hand control with their thoughts alone should also provide tremendous encouragement in the rehabilitation process.

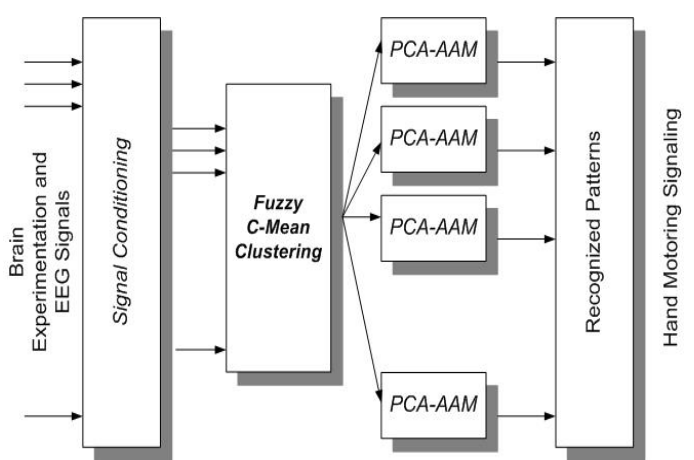

Fig. 3. System layers. Further details are found in Mattar et al. [11].

\section{EXPERIMENT: PATTERNS ACQUISITION}

Simultaneous EEG and hand kinematics were recorded from healthy participate, while they achieved a grasping task. The experimentations sessions were conducted to generate real-time signals. In reference to Luciw, et al. [15], twelve participates were involved, eight female, and the age was ranging between (19-35). This is further depicted in Fig. 4. Brain EEG patterns were recorded using special instrumentations. This involves the use of head-cup, measuring the waves patterns at various locations over the participants heads. In reference to Luciw, et al. [15], the EEG cap was BrainAmp sampled at $(5 \mathrm{kHz})$. The raw data were digitized, filtered, hence the data were stored. Data were stored in Matlab data structure format. This has made it easy to be processed a number of coding machines.

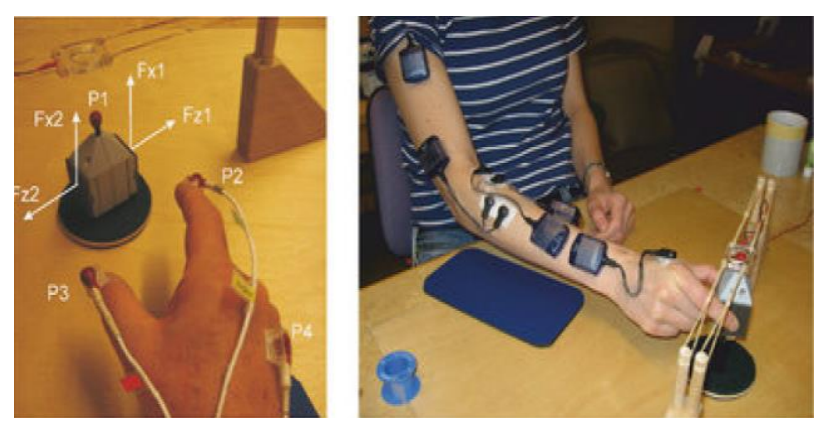

Fig. 4. Part of the grasping experimentations, Luciw, et al. [14].

\section{COMPUTATIONAL ANALYSIS}

\section{A. EEG Brainwaves Clustering Phase}

Clustering is referring to gather similar data patterns and behavior into within regions. These regions are also characterized by dimensionals $(x, y, z \ldots)$ and centers. Once dimensionals parameters and centers are detected from the EEG waves, then it becomes an easy task to associate any particular EEG wave pattern to a particular region. This is definitely useful for creating an association between the EEG brainwaves and the robotics-prosthesis hand. An important 
clustering paradigm is the fuzzy clustering. Fuzzy clustering is being characterized by the fuzziness of regions and boundaries, and the creation of (if then rules). Therefore, for detecting how the EEG patterns are related to the nature of the experiments (how EEG are related to motion of the fingers during the experimentation phase), we use fuzzy clustering (c-means) technique to compute such inherent signals associated patterns. Based on the above definitions, fuzzy clustering can be precisely formulated as an optimization problem. It involves a minimization of a defined functional in terms of distances from the clusters centers to each point within the EEG patterns. This is formulated as:

for each $i, k=\left(\begin{array}{llll}1 & 2 & \cdots & c\end{array}\right) \quad j=\left(\begin{array}{llll}1 & 2 & \cdots & n\end{array}\right)$, we need to minimize $J\left(u_{i j}, v_{k}\right)$ :

$$
J\left(u_{i j}, v_{k}\right)=\left\langle\sum_{i=1}^{c} \sum_{j=1}^{n} \sum_{k=1}^{c} g\left(w\left(x_{i}\right), u_{i j}\right) d\left(x_{j}, v_{k}\right)\right\rangle
$$

while observing the following constraints for $\left(\begin{array}{llll}j=1 & 2 & \cdots & n\end{array}\right)$

$$
\begin{gathered}
\left\langle\sum_{i=1}^{c} u_{i j}=1\right\rangle \\
\text { and }\left\langle 0 \leq \sum_{j=1}^{n} u_{i j} \leq n\right\rangle \text { for } i=\left(\begin{array}{llll}
1 & 2 & \cdots & c
\end{array}\right)
\end{gathered}
$$

One of the widely employed clustering methods based on Eq. (2) is the fuzzy $c$-means algorithm. The objective function of the FCM algorithm is expressed in the form of:

$$
J\left(u_{i j}, v_{k}\right)=\sum_{i=1}^{c} \sum_{j=1}^{n} u_{i j}^{m}\left\|x_{j}-v_{i}\right\|^{2}, \quad m>1
$$

In Eq. (3), ( $m$ ) is known as the exponential weight that influences the degree of fuzziness of the membership (partition) matrix.

\section{B. EEG Brainwaves Decoding Phase}

After we are done with the EEG waves partitioning via the clustering routine defined by Eq. (2) and $J\left(u_{i j}, v_{k}\right)$, we turn the attention to the primitive decoding methodology. This is correlation measure among the different patterns. Traditionally more rigorous statistical validation tests are employed in which the waves are examined, once found to be sufficiently correlated with a function of the data then the relation between waves can be measured. This is achieved by defining a matrix $\mathbf{Z}($.):

$$
\mathbf{Z}\left(\mathbf{x}^{t}\right)=\left(m(t), m(t-1), \ldots, m\left(t-t_{d}\right)\right)^{T}
$$

In Eq. (4), $\left(\mathbf{x}^{t}\right)$ is an observational vector of the EEG waves as (inputs), (outputs) and (errors), seen up to time step $(t)$ i.e.

$$
\mathbf{x}^{t}=\left(\mathbf{u}^{t-1}, \mathbf{y}^{t-1}, \mathbf{e}^{t-1}\right)^{T}
$$

and $m(t-1)$ is a monomial of the vector, as defined by:

$$
m(t)=\left(y(t-1) u^{2}(t-2)\right)
$$

The following two hypothesis can be defined:

$$
\begin{array}{ll}
H_{0}: & \mathbf{e}(t) \text { isuncorrelated with } \mathbf{Z}\left(\mathbf{x}^{t}\right), E(\mathbf{e}(t) \mid \mathbf{Z}(.))=0 \\
H_{1}: & \mathbf{e}(t) \text { isuncorrelated with } \mathbf{Z}\left(\mathbf{x}^{t}\right), E(\mathbf{e}(t) \mid \mathbf{Z}(.)) \neq 0
\end{array}
$$

and the purpose of validation is to use the data to decide if holds. Two different test statistics are employed, the most common being the standard sample correlation measure:

$$
\rho(k)=\left(\frac{1 / N \sum_{t=1}^{N-k+1}(m(t-k+1) \mathbf{e}(t))}{\sqrt{\sum_{t=1}^{N-k+1}(m(t-k+1) m(t-k+1)) \sum_{t=1}^{N-k+1}(\mathbf{e}(t) \mathbf{e}(t))}}\right)
$$

In Eq. (7), $k=1, \ldots, t_{d}$, and $\rho(k) \in[-1,1]$. If $H_{0}$ holds this statistic asymptotically approaches a normal distribution, and with $95 \%$ confidence limits $H_{0}$ is accepted if $\rho(k) \in[-1.96 / \sqrt{N}, 1.96 / \sqrt{N}]$.

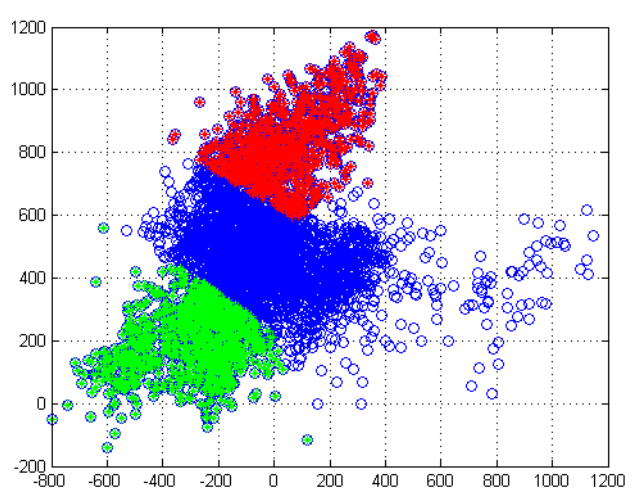

Fig. 5. Experimentations results. Three clustered patterns were detected. Further analysis is found in Mattar et al., [11].

Fuzzy clustering algorithm has been applied to the recorded EEG waves. Three clusters were therefore detected. This is shown in Fig. 5 and Fig. 6. This indicates that all of the recorded EEG patterns for a single try, at a particular time instant are nearly related to each other by similar patterns. This also indicates how such clusters are having overlapping regions.

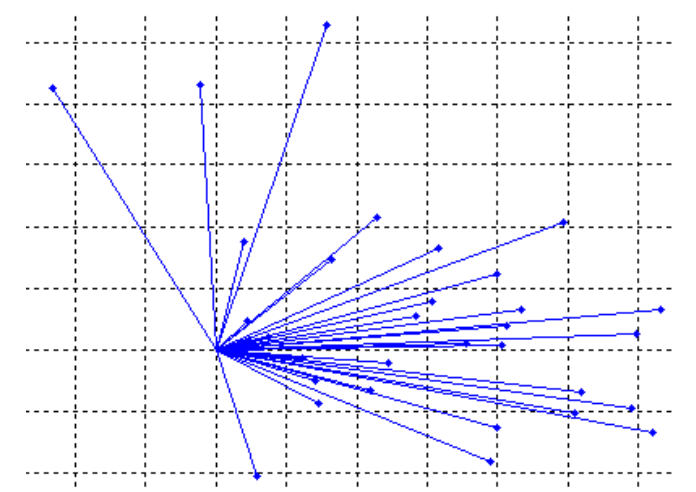

Fig. 6. Another representation of the clustered EEG patterns, vector representations. Further analysis is found in Mattar et al., [11].

\section{EEG-PATTERNS HAND ASSOCIATION}

In order to verify the adopted recognition approach, the system was simulated using the $e_{-}$Grasp. In reality, the $e_{-}$Grasp is a five fingers robotics hand simulator. $e_{-}$Grasp also runs on Matlab environment. It computes both the closed-chain kinematics, the closed chain-dynamics. It is also a fingers force optimization based-simulation environment. 
$e_{-}$Grasp was used to make use of the recorded related EEG patterns and data-set. Data patterns recorded from the EEG set were analysed, clustered, and recognized. Hence, an association was created between the EEG patterns, and the real fingers motions and gasping nature. The reason why $e \_$Grasp was adopted initially, this is because we would like to observe the hand fingers motion over the simulator, rather than real. Different run were conducted. This is including data for different robot hand grasping and behaviours. It is divided into two sets: TRAINING patterns, and VERIFICATION patterns. A number of behaviours of each Matlab-Based-simulation were used. Hence there are (3 simple hand behaviours) in the TRAINING phase and 3 behaviours over the VERIFICATION phase. Majority of hand behaviours run in the Matlab-Based-Simulation configuration data-set are for different grasp conditions. This is to create as much as possible of information about hand in space motion. $e_{-}$Grasp simulator has generated number of dissimilar hand behaviours (run over a number of times) compared with the hand behaviours data-set. This is tabulated in Table I.

TABLE I: GRASPING BEHAVIOURS AND ASSOCIATION

\begin{tabular}{|c|c|c|c|}
\hline Case & Behaviours & Human Behaviours & $\begin{array}{c}\text { Robotics Hand } \\
\text { Motoring }\end{array}$ \\
\hline$i$ & Light Grasp & $\begin{array}{c}\text { Human fingers are } \\
\text { closed around } \\
\text { object }\end{array}$ & $\begin{array}{c}\text { Robotic joints in finger } \\
\text { are motored in curling } \\
\text { shape }\end{array}$ \\
\hline \multirow{4}{*}{ ii } & Fair Grasp & $\begin{array}{c}\text { Human fingers are } \\
\text { closed around } \\
\text { object, with little } \\
\text { iii }\end{array}$ & $\begin{array}{c}\text { Hand joints are } \\
\text { motored in curling } \\
\text { shape, } \text { with force } \\
\text { control. }\end{array}$ \\
& Manipulation & $\begin{array}{c}\text { Human does fingers } \\
\text { motion, with } \\
\text { grasping and force } \\
\text { are in actions }\end{array}$ & $\begin{array}{c}\text { Hand joints are } \\
\text { motored in curling } \\
\text { shape, } \text { with continuous } \\
\text { control of motion and } \\
\text { force control. }\end{array}$ \\
\hline iv & $\begin{array}{c}\text { Fingers free } \\
\text { movements }\end{array}$ & $\begin{array}{c}\text { No fingers motion, } \\
\text { no grasping force } \\
\text { are in actions }\end{array}$ & $\begin{array}{c}\text { EEG } \text { waves and } \\
\text { patterns } \text { were detected }\end{array}$ \\
\hline
\end{tabular}

\section{CONCLUSION}

Learning EEG brainwaves resulting from grasping and manipulation mechanism was presented within this article. That was based on using human EEG brainwaves for training a robotics dexterous hand- prosthesis system. The learning approach was based on learning the inherent features and characterization for the wave patterns. Inherent characterizations were based on reducing the dimensionalities of the EEG waves, after performing clustering. The main issue that hinders better accuracy is the nature of such EEG waves, as they are totally related, correlated, and they are also of higher dimension, with almost random patterns. The presented methodology did reply on learning the gasping waves and patterns detected by a head cup. Due to the size of the detected EEG signals generated by the brain, pattern recognition technique was therefore adopted.

\section{ACKNOWLEDGMENT}

The author would like to sincerely express their appreciation and thanks to the Ume University, Ume, and to the researcher Luciw et al. [14], [15] for the helpful data.

\section{REFERENCES}

[1] S. Hatano, "Experience from a multicentre stroke register: A preliminary report," Bull World Health Organ, vol. 54, no. 5, pp. 541-553, 1976.

[2] C. Butefisch, H. Hummelsheim, P. Denzler, and K. H. Mauritz, "Repetitive training of isolated movements improves the outcome of motor rehabilitation of the centrally paretic hand," Journal Neurol Sci, vol. 130, pp. 59-68, 1995.

[3] J. R. Carey, T. J. Kimberley, S. M. Lewis, E. J. Auerbach, L. Dorsey, P. Rundquist, and K. Ugurbil, "Analysis of fMRI and finger tracking training in subjects with chronic stroke," Brain, vol. 125, pp. 773-788, 2002.

[4] E. Taub, N. E. Miller et al., "Technique to improve chronic motor deficit after stroke," Arch Phys Med Rehabil, vol. 74, pp. 347-354, 1993.

[5] B. Kopp, A. Kunkel, W. Muhlnickel, K. Villringer, E. Taub, and H. Flor, "Plasticity in the motor system related to therapy-induced improvement of movement after stroke," Neuroreport, vol. 10, pp. 807-810, 1999.

[6] C. E. Levy, D. S. Nichols, P. M. Schmalbrock, P. Keller, and D. W Chakeres, "Functional MRI evidence of cortical reorganization in upper-limb stroke hemiplegia treated with constraint-induced movement therapy," Am J Phys Med Rehabil, vol. 80, pp. 4-12, 2001.

[7] J. Liepert, I. Uhde, S. Graf, O. Leidner, and C. Weiller, "Motor cortex plasticity during forced-use therapy in stroke patients: A preliminary study," J. Neurol, vol. 248, pp. 315-321, 2001.

[8] H. Johansen-Berg, H. Dawes, C. Guy, S. M. Smith, D. T. Wade, and P. M. Matthews, "Correlation between motor improvements and altered fMRI activity after rehabilitative therapy," Brain, vol. 125, pp. 2731-242, 2002.

[9] C. E. Levy et al., "Functional MRI evidence of cortical reorganization in upper-limb stroke hemiplegia treated with constraint-induced movement therapy," Am J Phys Med Rehabil, pp. 4-12, 2001.

[10] K. Al Mutib, E. A. Mattar, and A. Al Mutib, "Learning grasping optimality with EEG hand recordings: BMI for robotic-prosthetic motion control," in Proc. $28^{\text {th }}$ International Conference on Computer Applications in Industry and Engineering, October 12-14, San Diego, California, USA, 2015.

[11] E. Mattar and K. Al-Mutib, "EEG complex grasping patterns recognition for BMI robotic and prosthesis motion control," in Proc. Michael Faraday IET International Summit, September 21-13, Kolkata, India, 2015.

[12] L. Carraro, "Promoting independence following a stroke: A guide for therapists and professionals working in primary health care," Geneva: Disability and Rehabilitation Team, WHO; AIFO, 1999.

[13] K. J Wineski et al., "Unique cortical physiology associated with ipsilateral hand movements and neuroprosthetic implications," Stroke, pp. 3351-3359, 2009.

[14] M. D. Luciw et al., "Multi-channel EEG recordings during 3,936 grasp and lift trials with varying weight and friction," Sci. Data, 2014.

[15] M. D. Luciw, E. Jarocka, and B. Edin. (2014). [Online]. Available: http://dx.doi.org/10.6084/m9.figshare.988376

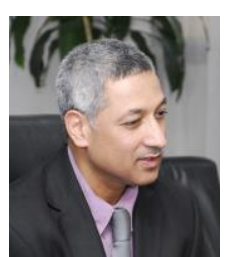

E. Mattar is Associate Professor of Cybernetics and Robotics of University of Bahrain, and Bahrain IET $\mathrm{LN} \mathrm{H}$. Chair. He is also a member of Bahrain NATIONAL Higher Education Skills-Innovation Steering Committee. Back on 1986, he received the bachelor of science in electrical eng. from University of Bahrain, pursued studies for MSc. in electronics in 1988 in University of Southampton, and in 1994, he was awarded a Ph.D. in cybernetics, robotics control from University of Reading with supervision of Prof. K. Warwick, professor of cybernetics. He worked on 13 research projects, including King Saud University Robotics Project, KSA. He supervised a number of (Ph.D., M.Sc., Undergraduate) students, and is currently working towards Brainwaves Decoding and Learning for robotics hand control. He got lots of awards, including University of Bahrain two best research awards in 1998, then 2001, 2002, 2006, and 2007 best undergraduate projects, Bahrain Police Academy Award in 2012, and others research related awards. Back in 2001 with 30 others from GCC, chosen for the Gulf Executive program in USA, hence he received the MBA from Darden, Virginia University. He has interest in cybernetics, robotics, computational AI, control, and lecturing within such areas. 